\section{Reinvestigation of the Synthesis of 2-Methylthio-trans-ribosylzeatin}

\author{
Tamizi Sugiyama, Mimiko Imura \\ and Takeshi HASHIZUME \\ Laboratory of Bioorganic Chemistry, Tokyo \\ Institute of Agriculture and Technology, \\ Fuchu, Tokyo 183, Japan
}

Received July 19, 1977

2-Methylthio-trans and cis-ribosylzeatin isolated from the plant tRNA were characterized by comparing the mass and ultraviolet spectra with those of their synthesized specimen. ${ }^{1,2}$ The trans isomer was synthesized in $18 \%$ yield by the condensation of 2,6-dimethylthio-9- $\beta$-D-ribofuranosylpurine and 4-hydroxy-3-methyl-trans-2-butenylamine. ${ }^{1)}$ The $c$ is isomer was prepared by the condensation of 2,6 -dichloro-9- $\beta$-D-ribofuranosylpurine and 4-hydroxy-3-methyl-cis-2-butenylamine, followed by treatment with sodium methyl mercaptide. ${ }^{2)}$ The yield was calculated to be $12.8 \%$. These methods, however, are unsuitable for synthesis of labeled cytokins which are required for the metabolic and quantitative studies. We, therefore, have reinvestigated the preparation of 6 -chloro-2-methylthio-9- $\beta$-D-ribofuranosylpurine ${ }^{3)}$ which is an important intermediate in the synthesis of the both isomers. This paper dealed with an improved synthetic method of 2-methylthio-transribosylzeatin from 6-chloro-2-methylthiopurine.

When 6-chloro-2-methylthiopurine ${ }^{4)}$ was fused with tetra-O-acetyl-D-ribofuranose ${ }^{b /}$ at $145 \sim 150^{\circ} \mathrm{C}$ for 20 $\mathrm{min}$ in the presence of concentrated sulfuric acid, three products (I, II and III) which gave a parent ion peak at $m / e 458$ were detected. These products were separated by silica-gel column chromatography and characterized after deacetylation.

Deacetylation of I, II and III affored IV, V and VI, respectively. Melting point of the compound VI agreed with that of authentic 6-chloro-2-methylthio- $9-\beta-D-$ ribofuranosylpurine, ${ }^{3)}$ and no depression was observed on admixing. The structure was also supported by the NMR and UV spectra.

The binding position of sugar moiety in the compound IV was assigned to be $\mathrm{N}-9$, as the UV spectra were similar to those of VI. ${ }^{3)}$ The anomeric configuration was assigned as $\alpha$ on the basis of NMR spectrum $\left(J_{1^{\prime} z^{\prime}}=9 \mathrm{~Hz}\right){ }^{\text {b) }}$ Thus, compound IV was determined as 6-chloro-2-methylthio-9- $\alpha$-D-ribofuranosylpurine.

The binding position of sugar moiety in the compound $V$ was also inferred to be $\mathrm{N}-9$, because the UV spectra were similar to those of IV and VI. ${ }^{3)}$ In order to determine further the binding position of sugar moiety, amination was performed to give 2-methylthio-9-pentosyladenine (VII) whose UV spectra were similar to those of 2-methylthioadenosine (VIII) ${ }^{\text {") }}$ It is unlikely that the sugar moiety of $\mathrm{V}$ is ribofuranose, since the admixture of $\mathrm{V}$ and VI showed a melting point depression. Thus, 6-chloro-2-methylthio-9- $\beta-\mathrm{D}$ ribopyranosylpurine (IX) was synthesized by the fusion method, but its physicochemical properties were not identical to those of $\mathrm{V}$. At present the compound $\mathrm{V}$ is assumed to be 6-chloro-2-methylthio-9-pentosylpurine since the nucleosides of 6-chloro-2-methylthiopurine were not available for identification.

This fusion procedure was originally reported by Sato who, however, characterized only VI. ${ }^{3}$ ) We now observed the formation of IV, V and VI in this reaction. Although these products were difficult to separate because of the close $R f$ value on tlc, their fully acetylated products (I, II and III) were able to separate.

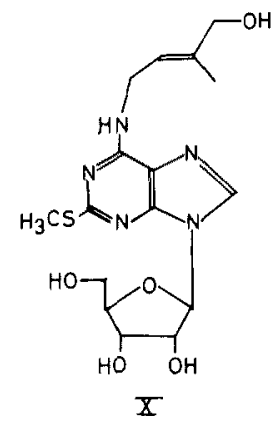

In this manner, 2-methylthio-trans-ribosylzeatin $(\mathrm{X})^{1)}$ was synthesized in $51.4 \%$ yield by the condensation of VI and 4-hydroxy-3-methyl-trans-2-butenylamine. ${ }^{3)}$ The NMR spectrum was in agreement with the structure assigned. The overall yield was approximately $14 \%$ which is almost twice as much as the conventional procedures, starting from 6-chloro-2-methylthiopurine.

\section{EXPERIMENTAL}

All mps were measured on a Yanagimoto meltingpoint apparatus. UV and NMR spectra were recorded with a Hitachi EPS-3T automatic spectrophotometer and a Hitachi R-24 instrument, respectively. MS spectra were obtained by a Shimadzu LKB 9000 , equipped with a direct inlet system.

Fusion of 6-chloro-2-methylthiopurine with tetra-Oacetyl-D-ribofuranose. A mixture of 6-chloro-2methylthiopurine ${ }^{4)}(1 \mathrm{~g})$ and tetra-O-acetyl-D-ribofuranose $e^{5)}(1.6 \mathrm{~g})$ was fused at $145 \sim 150^{\circ} \mathrm{C}$ for $20 \mathrm{~min}$ under the diminished pressure (water pump) in the presence of conc. $\mathrm{H}_{2} \mathrm{SO}_{4}(5 \mathrm{mg})$. The reaction mixture was extracted with chloroform. The chloroform was evaporated, and the residue was subjected to the silica- 
Table I. UV absorption Spectral Data of Trisubstituted Purines

\begin{tabular}{|c|c|c|c|}
\hline $\begin{array}{l}\text { Com- } \\
\text { pounds }\end{array}$ & $\begin{array}{c}0.1 \mathrm{~N}-\mathrm{HCl} \text { in } \\
95 \% \text { ethanol } \\
\lambda_{\max } \mathrm{nm} \\
\left(\varepsilon \times 10^{-3}\right)\end{array}$ & $\begin{array}{l}95 \% \text { ethanol } \\
\lambda_{\max } \mathrm{nm} \\
\left(\varepsilon \times 10^{-8}\right)\end{array}$ & $\begin{array}{c}0.1 \mathrm{~N}-\mathrm{NaOH} \\
\text { in } 95 \% \text { etha- } \\
\text { nol } \lambda_{\max } \mathrm{nm} \\
\left(\epsilon \times 10^{-3}\right)\end{array}$ \\
\hline & $235(17.1)$ & $235.5(17.6)$ & $265(14.2)$ \\
\hline \multirow[t]{3}{*}{ IV } & $264(11.9)$ & $264(11.9)$ & $285^{\circ}(9.8)$ \\
\hline & $307(7.9)$ & $307(7.8)$ & $305^{s}(8.0)$ \\
\hline & $236.5(16.4)$ & $236(17.5)$ & $262(16.8)$ \\
\hline \multirow[t]{3}{*}{ V } & $264(11.2)$ & $264(11.2)$ & $282^{\mathrm{s}}(13.4)$ \\
\hline & $307(8.1)$ & $307(7.4)$ & $306^{8}(9.5)$ \\
\hline & $238(17.4)$ & $238(18.0)$ & $264(12.8)$ \\
\hline \multirow[t]{2}{*}{ VI } & $265.5(11.0)$ & $265(11.0)$ & $285^{\mathrm{s}}(13,4)$ \\
\hline & $307(7.4)$ & $307(7.4)$ & $306^{s}(6.9)$ \\
\hline \multirow[t]{2}{*}{ VII } & $270(14.2)$ & $235.5(21.0)$ & $276(17.4)$ \\
\hline & & $276(14.5)$ & \\
\hline \multirow[t]{3}{*}{$\mathrm{VIII}^{3,7)}$} & $270(16.0)$ & $237(24.3)$ & $235(21.2)$ \\
\hline & & $277(14.5)$ & $277(14.7)$ \\
\hline & $235(16,1)$ & $235.5(17.0)$ & $264(22.3)$ \\
\hline \multirow[t]{2}{*}{ IX } & $265(12.2)$ & $265(11.7)$ & $282^{3}(18.8)$ \\
\hline & $307(7.9)$ & $308(7.4)$ & $305^{8}(11.7)$ \\
\hline
\end{tabular}

$\mathrm{s}=$ shoulder

gel column $(2.1 \times 40 \mathrm{~cm})$. The column was eluted stepwise with $13 \%$ (1.5 liter), $15 \%$ (0.5 liter), $17 \%$ (0.5 liter) and $19 \%(0.5$ liter $)$ ethyl acetate in benzene. The effluent was collected in $20 \mathrm{ml}$ fractions and examined by analytical tlc. I and III were obtained from the fractions $37 \sim 49$ and $59 \sim 120$, respectively. II was isolated from the fractions $50 \sim 58$ containing trace amount of III by preparative tlc.

I. Yield, $335 \mathrm{mg}(14.6 \%)$. MS $m / e 458\left(\mathrm{M}^{+}\right)$. UV $\lambda_{\max }^{\mathrm{EtOH}} 265 \mathrm{~nm}$. NMR $\left(\mathrm{CDCl}_{3}\right) \delta: 8.24\left(1 \mathrm{H}, \mathrm{s}, \mathrm{C}_{\beta}-\mathrm{H}\right)$, $2.64\left(3 \mathrm{H}, \mathrm{s}, \mathrm{SCH}_{3}\right), 1.83,2.05,2.27(3 \mathrm{H}, 3 \mathrm{H}, 3 \mathrm{H}, \mathrm{s}$, acetyl protons). $R f, 0.32$ (benzene: ethyl acetate $=2: 1$, $\mathrm{v} / \mathrm{v})$.

II. Yield, $149 \mathrm{mg}(6.5 \%)$. MS $m / e 458\left(\mathrm{M}^{+}\right)$. UV $\lambda_{\max }^{\mathrm{EtoH}} 262.5 \mathrm{~nm}$. NMR $\left(\mathrm{CDCl}_{\mathrm{g}}\right) \delta: 8.23\left(1 \mathrm{H}, \mathrm{s}, \mathrm{C}_{8}-\mathrm{H}\right)$, $2.58\left(3 \mathrm{H}, \mathrm{s}, \mathrm{SCH}_{3}\right), 1.85,2.05,2.13(3 \mathrm{H}, 3 \mathrm{H}, 3 \mathrm{H}, \mathrm{s}$, acetyl protons). $R f, 0.28$ (benzene: ethyl acetate $=$ 2: $1, \mathrm{v} / \mathrm{v})$.

III. Yield, $882 \mathrm{mg}(38.5 \%)$. MS $m / e 458\left(\mathrm{M}^{+}\right)$. UV $\lambda_{\mathrm{max}}^{\mathrm{EtOH}} 264.5 \mathrm{~nm}$. NMR $\left(\mathrm{CDCl}_{3}\right) \delta: 8.17\left(1 \mathrm{H}, \mathrm{s}, \mathrm{C}_{8}-\mathrm{H}\right)$, $2.63\left(3 \mathrm{H}, \mathrm{s}, \mathrm{SCH}_{3}\right), 2.03,2.08,2.13(3 \mathrm{H}, 3 \mathrm{H}, 3 \mathrm{H}, \mathrm{s}$, acetyl protons). $R f, 0.21$ (benzene: ethyl acetate $=$ $2: 1, v / v)$.

6-Chloro-2-methylthio-9-a-D-ribofuranosylpurine (IV). I was dissolved in methanolic ammonia $(50 \mathrm{ml})$. The solution was kept at $4^{\circ} \mathrm{C}$ for overnight, followed by removal of the solvent. The residue was crystallized from ethanol to give colorless needles. Yield, $162 \mathrm{mg}$ $\left(66.4 \%\right.$ ). mp, $248 \sim 252^{\circ} \mathrm{C}$. NMR (DMSO-d $\mathrm{d}_{6}+\mathrm{D}_{2} \mathrm{O}$ ) $\delta: 8.53\left(1 \mathrm{H}, \mathrm{s}, \mathrm{C}_{8}-\mathrm{H}\right), 5.67\left(1 \mathrm{H}, \mathrm{d}, J=9 \mathrm{~Hz}, \mathrm{C}_{1}-\mathrm{H}\right)$, $2.57\left(3 \mathrm{H}, \mathrm{s}, \mathrm{SCH}_{3}\right)$. MS m/e $332\left(\mathrm{M}^{+}\right)$. Anal. found:
C, 39.96; $\mathrm{H}, 3.99 ; \mathrm{N}, 17.18$; calcd. for $\mathrm{C}_{11} \mathrm{H}_{13} \mathrm{O}_{4} \mathrm{~N}_{4} \mathrm{SCl}$ : C, $39.69 ; \mathrm{H}, 3.91 ; \mathrm{N}, 16.84 \%$. Rf, 0.19 (ethyl acetate: ethanol $=10: 1, \mathrm{v} / \mathrm{v})$.

6-Chloro-2-methylthio-9-pentosylpurine (V). Deacetylation of II in $40 \mathrm{ml}$ of methanolic ammonia yielded $\mathrm{V}$, as described above. Yield, $65 \mathrm{mg}(61.7 \%)$. mp, 205 208 ${ }^{\circ} \mathrm{C}$. NMR (DMSO- $\mathrm{d}_{8}+\mathrm{D}_{2} \mathrm{O}$ ) $\delta: 8.63$ $\left(1 \mathrm{H}, \mathrm{s}, \mathrm{C}_{8}-\mathrm{H}\right), 6.44\left(1 \mathrm{H}, \mathrm{d}, J=6 \mathrm{~Hz}, \mathrm{C}_{1} \cdot-\mathrm{H}\right), 2.62(3 \mathrm{H}$, $\left.\mathrm{s}, \mathrm{SCH}_{3}\right)$. MS m/e $332\left(\mathrm{M}^{+}\right)$. Anal. found: C, 39.59; $\mathrm{H}, 3.94 ; \mathrm{N}, 16.56$; calcd. for $\mathrm{C}_{11} \mathrm{H}_{13} \mathrm{O}_{4} \mathrm{~N}_{4} \mathrm{SCl}$ : C, 39.69; $\mathrm{H}, 3.91 ; \mathrm{N}, 16.84 \% . \quad R f, 0.30$ (ethyl acetate: ethanol= $10: 1, \mathrm{v} / \mathrm{v})$.

6-Chloro-2-methylthio-9- $\beta$-D-ribofuranosylpurine (VI). III was deacetylated with methanolic ammonia $(50 \mathrm{ml})$ to give VI. Recrystallization from ethanol gave a pure product. Yield, $448 \mathrm{mg}(70 \%) . \mathrm{mp}, 183 \sim 184^{\circ} \mathrm{C}$ (lit; $181^{\circ} \mathrm{C}^{5)}$ ). NMR (DMSO- $\left.\mathrm{d}_{\beta}+\mathrm{D}_{2} \mathrm{O}\right) \delta: 8.70(1 \mathrm{H}, \mathrm{s}$, $\left.\mathrm{C}_{8}-\mathrm{H}\right), 5.97\left(1 \mathrm{H}, \mathrm{d}, J=6 \mathrm{~Hz}, \mathrm{C}_{1}-\mathrm{H}\right), 2.60\left(3 \mathrm{H}, \mathrm{s}, \mathrm{SCH}_{8}\right)$. MS m/e $332\left(\mathrm{M}^{+}\right)$. Anal. found: C, $39.81 ; \mathrm{H}, 4.01 ; \mathrm{N}$, 16.86 ; calcd. for $\mathrm{C}_{11} \mathrm{H}_{18} \mathrm{O}_{4} \mathrm{~N}_{4} \mathrm{SCl}: \mathrm{C}, 39.69 ; \mathrm{H}, 3.91 ; \mathrm{N}$, $16.84 \% . R f, 0.31$ (ethyl acetate: ethanol $=10: 1, \mathrm{v} / \mathrm{v}$ ).

2-Methylthio-9-pentosyladenine (VII). A solution of $\mathrm{V}(20 \mathrm{mg})$ in methanolic ammonia $(5 \mathrm{ml})$ was heated in a sealed tube at $100^{\circ} \mathrm{C}$ for $5 \mathrm{hr}$. The reaction mixture was evaporated to dryness, and the residue was crystallized from ethanol. Yield, $8 \mathrm{mg}(42 \%$. $\mathrm{mp}, 222 \sim$ $223^{\circ} \mathrm{C}$. MS $m / e 313\left(\mathrm{M}^{+}\right)$. Anal. found: $\mathrm{C}, 42.76 ; \mathrm{H}$, $4.77 ; \mathrm{N}, 35.36$; calcd. for $\mathrm{C}_{11} \mathrm{H}_{15} \mathrm{O}_{4} \mathrm{~N}_{5} \mathrm{~S}: \mathrm{C}, 43.08 ; \mathrm{H}$, $4.62 ; \mathrm{N}, 35.90 \%$.

6-Chloro-2-methylthio-9- $\beta$-D-ribopyranosylpurine (IX). 6-Chloro-2-methylthiopurine ${ }^{4)}(0.4 \mathrm{~g})$ was fused with tetra-O-acetyl-D-ribopyranose $\mathrm{b}^{\mathrm{b})}(0.7 \mathrm{~g})$ at $145 \sim 150^{\circ} \mathrm{C}$ for $30 \mathrm{~min}$ in the presence of conc. $\mathrm{H}_{2} \mathrm{SO}_{4}(3 \mathrm{mg})$. The reaction mixture was extracted with chloroform. The chloroform was evaporated, and residue was subjected to silica-gel column $(2.1 \times 40 \mathrm{~cm})$. The column was eluted with benzene: ethyl acetate $(5: 1$, $\mathrm{v} / \mathrm{v})$. The effluent was collected in $20 \mathrm{ml}$ fractions and examined by analytical tlc. The fractions containing product were combined and evaporated to dryness. The residue was dissolved in methanolic ammonia. The solution was kept at $4^{\circ} \mathrm{C}$ for overnight, followed by removal of the solvent. The residue was crystallized from ethanol. Yield, $178 \mathrm{mg}(27 \%) . \mathrm{mp}, 239 \sim 246^{\circ} \mathrm{C}$. NMR (DMSO-d $\left.\mathrm{d}_{\mathrm{B}}+\mathrm{D}_{2} \mathrm{O}\right) \delta: 8.65\left(1 \mathrm{H}, \mathrm{s}, \mathrm{C}_{\mathrm{B}}+\mathrm{H}\right), 5.71$ $\left(1 \mathrm{H}, \mathrm{d}, J=8 \mathrm{~Hz}, \mathrm{C}_{1},-\mathrm{H}\right), 2.60\left(3 \mathrm{H}, \mathrm{s}, \mathrm{SCH}_{3}\right)$. MS mle $332\left(\mathrm{M}^{+}\right)$. Anal. found: C, 39.71; H, 3.98; N, 16.41; calcd. for $\mathrm{C}_{11} \mathrm{H}_{13} \mathrm{O}_{4} \mathrm{~N}_{4} \mathrm{SCl}: \mathrm{C}, 39.69 ; \mathrm{H}, 3.91 ; \mathrm{N}$, $16.84 \%$

2-Methylthio-trans-ribosylzeatin (X) [6-(4-hydroxy-3methyl-trans - 2-butenylamino)-2-methylthio-9-3-D-ribofuranosylpurine]. A mixture of VI (100 mg), 4hydroxy-3-methyl-trans-2-butenylamine $e^{8)}(150 \mathrm{mg})$ and 
triethylamine $(500 \mathrm{mg})$ in $n$-butanol $(10 \mathrm{ml})$ was refluxed for $4 \mathrm{hr}$. After cooling, the reaction mixture was evaporated to dryness under the diminished pressure. The residue was subjected to the silica-gel column $(1.9 \times$ $22 \mathrm{~cm}$ ). The column was eluted with a solution of ethyl acetate and ethanol $(10: 1, v / v)$. The fractions containing product were combined and evaporated to dryness. The residue $(101 \mathrm{mg})$ was crystallized from $50 \%$ aqueous ethanol to give colorless needles. Yield, $61 \mathrm{mg}\left(51.4 \%\right.$ ). mp, 154.5 157 $\mathrm{C}$ (lit. $155 \sim 156^{\circ} \mathrm{C}^{1)}$ ). MS m/e $397\left(\mathrm{M}^{+}\right)$. Anal. found: $\mathrm{C}, 48.57 ; \mathrm{H}, 5.82$; $\mathrm{N}, 17.58$; calcd. for $\mathrm{C}_{10} \mathrm{H}_{28} \mathrm{O}_{5} \mathrm{~N}_{5} \mathrm{~S}: \mathrm{C}, 48.36 ; \mathrm{H}, 5.79$; $\mathrm{N}, 17.63 \%$.

\section{REFERENCES}

1) W. J. Burrows, D. J. Armstrong, M. Kaminek, F. Skoog, F. M. Bock, S. M. Hecht, L. G. Damman, N. J. Leonard and J. Occolowitz, Bio- chemistry, 9, 1867 (1970).

2) H. J. Verman, R. Y. Schmitz, F. Skoog, A.J. Playtis, C. R. Frihart and N. J. Leonard, Phytochemistry, 13, 31 (1974).

3) T. Sato, "Synthetic Procedures in Nucleic Acid Chemistry," Vol. 1, ed. by W. W. Zorbach and R. S. Tipson, Wiley-Interscience, 1968, p. 264.

4) C. W. Noell and R. K. Robins, J. Org. Chem., 24, 320 (1959).

5) G. B. Brown, J. Davoll and B. A. Lowy, Biochem. Prepn., 4, 70 (1955).

6) L. B. Townsend, "Synthetic Procedures in Nucleic Acid Chemistry," Vol. 2, ed. by W. W. Zorbach and R.S. Tipson, Wiley-Interscience, 1973, p. 267.

7) J. Davoll and B. A. Lowy, J. Am. Chem. Soc., 74, 1563 (1952).

8) G. Show, B. M. Smallwood and D. V. Wilson, $J$. Chem. Soc., 1966, 921. 